\title{
Soliton-Like Waves in the Vicinity of the Southern Kuril Islands
}

\author{
V. A. Squire ${ }^{1}$, P. D. Kovalev ${ }^{2}$, D. P. Kovalev ${ }^{2}$ \\ ${ }^{1}$ Department of Mathematics and Statistics, University of Otago, Dunedin, New Zealand \\ ${ }^{2}$ Institute of Marine Geology and Geophysics, Far East Branch of \\ Russian Academy of Sciences, Yuzhno-Sakhalinsk, Russian Federation \\ 凶kovalev_pd@outlook.com
}

Purpose. This paper focuses on modulated solitons detected in time series of observational data on sea level oscillations in the Sea of Okhotsk, verifying the presence of nonstationary processes within a quantitative framework of methods.

Methods and Results. The paper reports an analysis of wave observation data collected using ARWtype, bottom-mounted pressure sensors in the area of the Capes Castricum, Van-der-Lind and Lovtsova in the southern Kuril Islands. The time series obtained were bandpass filtered using hardware with a passband of 20 minutes to 2.5 hours. Residual time series show the presence of wave packets generated at the beginning of the $\mathrm{K}_{1}$ (diurnal) tide, which consistently appear as a group consisting of 5-7 packets. It is shown that the synchronicity between each wave packet and the $\mathrm{K}_{1}$ wave initiation is associated with the cyclic separation of the tidal flow of the $\mathrm{K}_{1}$ oscillation at the elevation in the Urup Strait located between the islands, along with a concomitant spawning of vortices. It is speculated that the vortices generate the detected wave packets, which are each found to encase a cluster of waves with an average period of about 1.6 hours that are attributed to either edge waves or shelf seiches or a combination of both. A numerical model simulation of the detected wave packets was performed using the Korteweg - de Vries equation, confirming that the envelope of the observed wave packets is close to the modeled one and behaves like a soliton.

Conclusions. It is shown that synchronous initiation of a wave packet and a $\mathrm{K}_{1}$ wave is associated with the cyclic separation of the tidal flow of the $\mathrm{K}_{1}$ oscillations at a subsurface elevation in the Urup Strait located between the islands, with a concomitant spawning of vortices. The vortices are assumed to generate the detected wave packets. Each packet contains a cluster of waves with an average period of about 1.6 hours, which is conditioned by the period of the edge wave or shelf seiche. Spectral analysis performed for the 4.5-day-long time series with and without the groups of solitons, showed that the wave energy increases in the $0.5-5.5$ hour period range when solitons occur. Application of a simple amplitude-based criterion permitted the authors to identify the waves detected in the wave packets as anomalous. Transformation of the time series into normalized time and normalized amplitude coordinates show that all the examples of anomalous wave packets could be modeled using the Korteweg - de Vries time equation.

Keywords: Sea of Okhotsk, soliton-like waves, Korteweg - de Vries equation, solitons, harmonic breathers

Acknowledgements: P. D. Kovalev and D. P. Kovalev performed this investigation in accordance with the state program of IMGG-FEB-RAS. They thank the staff of the Laboratory of Wave Dynamics and Coastal Currents for collecting the field data. V. A. Squire highly appreciates the continued support from the University of Otago throughout his long scientific career and especially acknowledges fruitful collaboration with the graduate students and the postdoctoral fellows.

For citation: Squire, V.A., Kovalev, P.D. and Kovalev, D.P., 2021. Soliton-like Waves in the Vicinity of the Southern Kuril Islands. Physical Oceanography, [e-journal] 28(1), pp. 3-19. doi:10.22449/0233-7584-2021-1-3-19

DOI: $10.22449 / 0233-7584-2021-1-3-19$

(C) V. A. Squire, P. D. Kovalev, D. P. Kovalev, 2021

(C) Physical Oceanography, 2021

Introduction. The Institute of Marine Geology and Geophysics of the Far Eastern Branch of the Russian Academy of Sciences (IMGiG FEB RAS) has been 
studying marine phenomena such as tsunamis, meteotsunami, storm surges and waves in the coastal zones of Sakhalin Island and the Kuril Islands for many years. In 2008-2009 wave recordings were made in the vicinity of Cape Lovtsova at the northeastern part of Kunashir Island and near Capes Van der Lind and Castricum on the southwestern and northeastern ends of Urup Island, with the purpose of monitoring the natural oscillations within areas of water nearby that could potentially contribute to the strengthening of incident tsunami waves. Field observations were conducted using autonomous ARW-series seafloor-mounted wave recorders, which are sensitive across a wide range of periods from $2 \mathrm{~s}$ to tidal. The duration of each recorded time series varied from 4 to 6 months.

The time series of sea level fluctuations obtained were filtered to emphasize waves in the period bands associated with tsunamis and meteotsunamis. Preprocessing was done with a bandpass filter with a bandwidth from 20 min to $2.5 \mathrm{~h}$. Visual analysis of the filtered time series of observations in the area of Cape Castricum revealed wave packets covering waves with a period of about $1.6 \mathrm{~h}$. The wave packets occurred in groups of approximately 6 waves and appear to be synchronized with the beginning of the $K_{1}$ (diurnal) tide. It is conjectured that the detected waves can be attributed to soliton-like oscillations or breathers [1-6], in which the oscillations are modulated within a soliton-like envelope. In work [6, p. 9759], the definition of soliton-like waves and breathers is given: “... these are nonlinear modes existing in a wide range of physical systems. They are fundamental solutions to a number of nonlinear wave evolution equations, including the unidirectional nonlinear Schrödinger equation”.

A number of studies (e.g., [7], carried out using measurements in Bussol Strait in the Kuril Islands in the vicinity of our observations and, more generally, works $[8,9])$, discuss the synchronicity of internal solitary waves and tides. Such phenomena have been monitored in many places around the globe using a variety of field and remote sensors, such as synthetic aperture radars installed on satellites and aircraft. In addition, in [10] it is shown that the instability which occurs when flows separate from submarine elevations between islands is characterized by pulsating processes in stagnant zones. The mechanism of these processes has not yet been described theoretically, so currently the frequency and size of pulsating vortices can only be determined experimentally. Contemporary authors postulate that these pulsating vortices can cause wave packets to form that associate with the $\mathrm{K}_{1}$ tide, which is confirmed in the current work.

The Korteweg - de Vries (KdV) equation or its modifications are often used to establish the relationship between soliton-like waves and the types of wave packets described herein, especially when the water column is relatively shallow compared to the wavelengths observed. This equation was introduced by J. V. Boussinesq in 1877, but acquired its modern form in the works of Diederik Korteweg and Gustav de Vries, who reinterpreted it, investigated it more fully [11] and obtained a nonlinear equation for describing long solitary waves on water. To study the spatial evolution of the sea surface level measured at a certain point, the KdV equation can be used in the so-called temporal form (given in [12]), with an initial disturbance at this point. However, the equation in [12] describes only the soliton wave envelope, which coincides with the envelope of the observed packets. In the present case, wave packets which take the form of modulated oscillations are recorded. For such waves, the given time series can be compared with theoretical predictions based upon the nonlinear harmonic breather method introduced in [3]. 
According to this method, various wave packets are decomposed into their fundamental harmonic components by applying the Fourier series transformation built on trigonometric basis functions of linear theory. The frequent presence of soliton waves in shallow sea zones was also noted in [13-15] and a number of other papers. Because we detected modulated solitons in the sea level time series recorded by the IMGiG FEB RAS, it is of interest to check for the presence of these hydrophysical processes within the framework of quantitative methods.

Data of observations. Observations of waves over a wide range of periods were carried out in the vicinity of the South Kuril Islands of the volcanic archipelago in the Sakhalin region of Russia, stretching for about $1300 \mathrm{~km}$ northeastward from Hokkaido to Kamchatka and separating the Sea of Okhotsk from the northern part of the Pacific Ocean. Wave recorders were installed near Capes Castricum and Van der Lind on Urup Island at depths of about 15 and $21 \mathrm{~m}$, respectively. The third recorder was installed in the area of Cape Lovtsova at a depth of about $25 \mathrm{~m}$. The bottom hydrostatic pressure was measured and then converted into sea level fluctuations, i.e., waves on the sea surface, taking into account the attenuation of the shorter waves with depth.

The location of the installed recorders is shown in Fig. 1. The distance between Cape Castricum and Cape Van der Lind is approximately $114 \mathrm{~km}$, and between Cape Van der Lind and Cape Lovtsova is about $250 \mathrm{~km}$. The duration of each time series recorded is roughly 115 days, whilst the data were sampled every second.

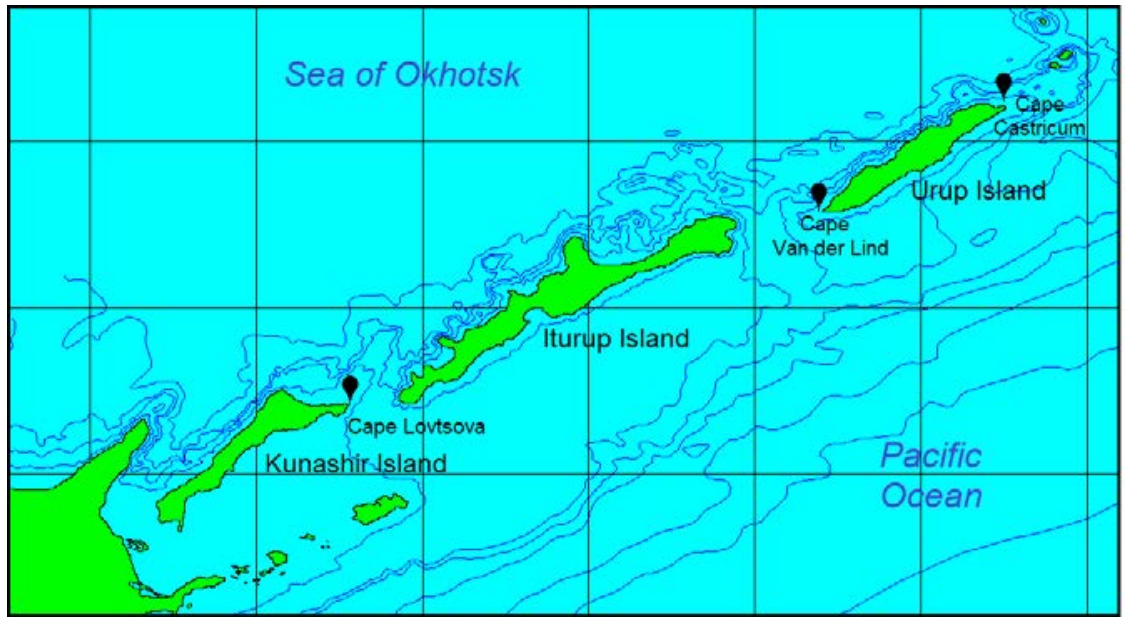

F i g. 1. Map of the observation region and the locations of the devices

The time series obtained at the observation points of Cape Castricum, Cape Van der Lind and Cape Lovtsova contained significant short-period noise, although the oscillations caused by wind waves and swell in the original data set had amplitudes of no more than $20 \mathrm{~cm}$ unless a storm was present. Regardless of this, each time series of observations was filtered so as to exclude noise outside the passband from 20 min to 2.5 hours. After filtering of the data, the times were found when increases occurred in the amplitude of the waves attributed to single wave packets, taking into account the conclusions on the separation of single PHYSICAL OCEANOGRAPHY VOL. 28 ISS. 1 (2021) 
waves set out in the thesis [13]. The time scales of the waves observed in the present study also turned out to be close to those given in that work.

Strictly speaking, interpreting a random wave field in shallow water in terms of Fourier spectra is unsatisfactory when the wave amplitudes are not small. Nonlinearity of wave fields leads to harmonic interactions and random changes in the Fourier spectra [12]. Taking into account this prerequisite, but also recognizing that the amplitudes of the detected waves are modest and nonlinearity only appears sporadically, the authors decided to accept the use of Fourier spectra for sea level fluctuation analysis.

Both the original recordings and the processed time series (Fig. 2) show anomalous waves. Several easily-distinguishable packets contain waves with periods of about 1.6 hours, evident within the series that were filtered to eliminate short-period noise. The wave amplitudes within the packets varied from 5 to $25 \mathrm{~cm}$, whilst the maximum duration of the entire packet is usually about 4.7 hours.

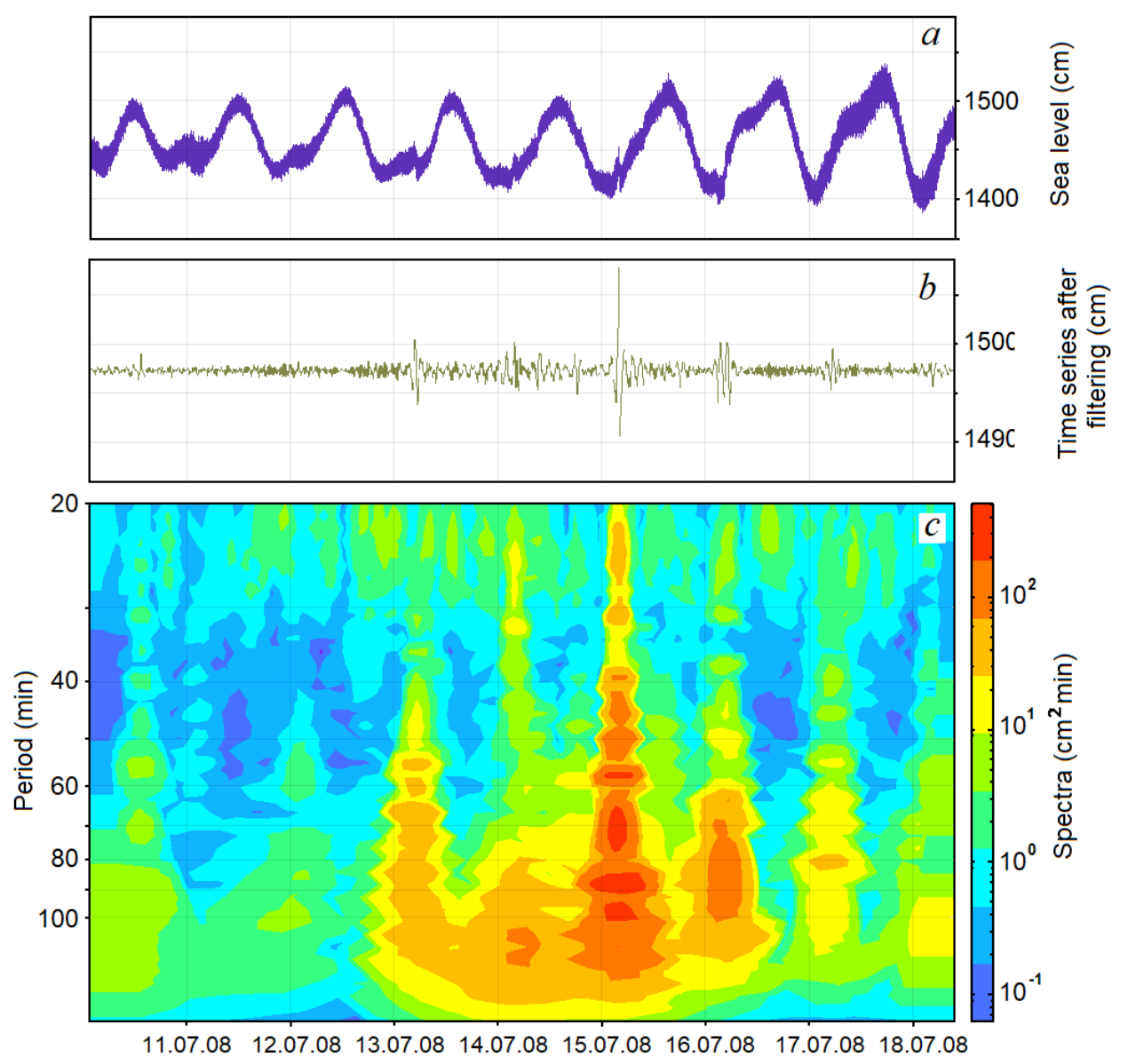

F i g. 2. A fragment of the recorded time series, July 11-18, 2008 (a); after bandpass filtering in the period range 20 minutes -2.5 hours $(b)$, and its spectral density $(c)$ 
Initially, in the visual analysis of each time series, single wave packets with amplitudes exceeding $10 \mathrm{~cm}$ were selected. Later it was found that wave packets actually appear in groups where the wave amplitude increases from packet to packet and then returns to its background level. Accordingly, a significant amplitude was determined subsequently to be one above the background level. The calculated data for various fragments of observations in the form of 2-day segments are presented in the table.

Mean value of the sea level $z(\mathrm{~cm})$, mean square deviation $\sigma(\mathrm{cm})$, skewness $S k$ and kurtosis $K u$ coefficients, significant wave amplitude $A_{s}(\mathrm{~cm})$ and anomalous amplitude $A_{a b}(\mathrm{~cm})$

\begin{tabular}{c|cc|c|c|c|c|c}
\hline & $\begin{array}{c}\text { Start date of the } \\
\text { No-day series in } \\
2008\end{array}$ & $Z$ & $\sigma$ & $S k$ & $K u$ & $A_{s}$ & $A_{a b}$ \\
\hline 1 & July, 11 & 1494.48 & 1.76 & 0.05 & 3.08 & 3.52 & 7.04 \\
\hline $\mathbf{2}$ & July, 14 & $\mathbf{1 4 9 4 . 4 8}$ & $\mathbf{4 . 1 6}$ & $\mathbf{1 . 5 4}$ & $\mathbf{2 6 . 1 3}$ & - & - \\
\hline 3 & August, 6 & 1494.48 & 1.71 & 0,04 & 4.13 & 3.41 & 6.82 \\
\hline $\mathbf{4}$ & August, 11 & $\mathbf{1 4 9 4 . 4 8}$ & $\mathbf{3 . 3 0}$ & $-\mathbf{0 . 2 0}$ & $\mathbf{7 . 6 8}$ & - & - \\
\hline 5 & \begin{tabular}{c} 
September, 3 \\
\hline $\mathbf{6}$
\end{tabular} & 1494.48 & 2.13 & -0.09 & 3.06 & 4.26 & 8.52 \\
\hline 7 & September, 7 & $\mathbf{1 4 9 4 . 4 8}$ & $\mathbf{3 . 9 4}$ & $-\mathbf{0 . 1 0}$ & $\mathbf{7 . 1 2}$ & - & - \\
\hline $\mathbf{8}$ & October, 20 & 2423.18 & 2.02 & -0.06 & 3.17 & 4.04 & 8.08 \\
\hline Average rating for no wave packets & 1.90 & -0.01 & 3.36 & 3.81 & 7.62 \\
\hline /Average scores when wave packets & $\mathbf{3 . 9 3}$ & $-\mathbf{0 . 1 4}$ & $\mathbf{1 2 . 5 3}$ & - & - \\
\hline
\end{tabular}

Note: Bold text indicates parameters for time series containing registered wave packets. Series 1-8 refer to the observations at Cape Castricum, series 9 and 10 to the observations at Cape Lovtsova

Spectral analysis carried out for 4.5-day time series with and without wave packets shows (Fig. 3) that, when wave packets are present in the record, the wave energy increases in the range of periods of 0.5-5.5 hours. All wave packets in groups correspond to the start of the tide. During 115 days of measurements in the area of Cape Castricum, four separate groups of waves and one group of waves corresponding to the minimum water level but observed during a semidiurnal high tide near Cape Lovtsova, were recorded. Near Cape Van der Lind, no waves with periods close to 1.6 hours were recorded. 


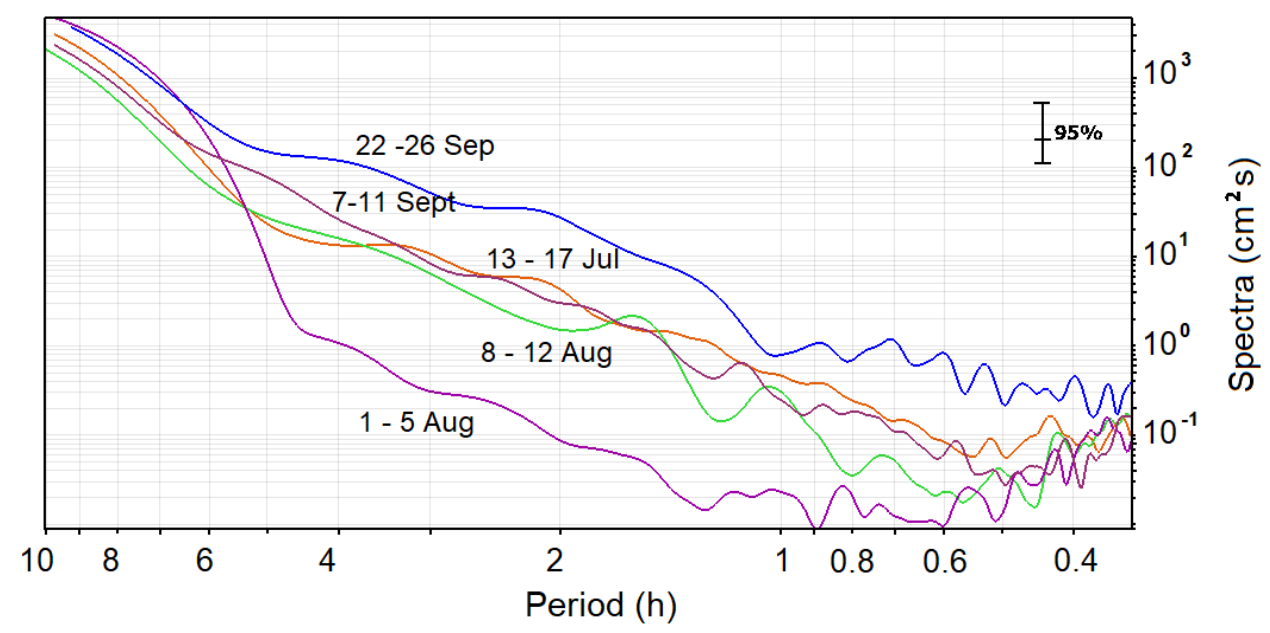

F i g. 3. Power density spectra for 4.5-day-long time series including a group of wave packets (22-26 September time series - blue curve; 7-11 September time series - deep-purple curve; 13-17 July time series - brown curve; 8-12 August time series - green curve) and in their absence (1-5 August time series - purple curve)

Wave packets. To determine the necessary conditions for the formation of the wave packets that were observed, the weather conditions at their time of appearance and the bathymetric features of the coast where they were observed were studied. It was found that the height of the wind waves and swell at the moments of observation of the wave packets usually did not exceed $20 \mathrm{~cm}$ for Cape Castricum and $40 \mathrm{~cm}$ for Cape Lovtsova, with the exception of aberrant waves that reached $79 \mathrm{~cm}$. Wave packets were also observed in calm weather in the area, except at Cape Lovtsova. This implies that sea state did not affect the generation of wave packets.

The fact that there were no wave packets near Cape Van der Lind and only a single example was recorded near Cape Lovtsova is probably due to the bathymetric features of the straits between the islands. On the ocean shelf diagram (Fig. 4), taken from [16, p. 229], Cape Castricum is located in the southern part of Urup Strait (profile 3 in Fig. 4, b). The strait depth is about 500 m, which is less than half the width of the $40 \mathrm{~km}$-wide Vries Strait (between Iturup and Urup Islands). The maximum depth of the Vries Strait is about $1300 \mathrm{~m}$ (profile 2, Fig. 4, $b$ [17, p. 87]); Cape Van der Lind is located there. Meanwhile, in the Cape Lovtsova area (profile 1, Fig. 4, b), the approximately 21-km-wide Catherine Strait between Iturup and Kunashir islands has a depth of about $400 \mathrm{~m}$ - almost the same as the Urup Strait of $30 \mathrm{~km}$ width. Thus, the depth of the sea is apparently the governing parameter for the generation of the wave packets that are observed in the shallower regions of the Catherine Strait and the Urup Strait. It is also possible that the projection of the coastal shelf, clearly visible on profiles 1 and 3 (Fig. 4), plays a specific role here. 

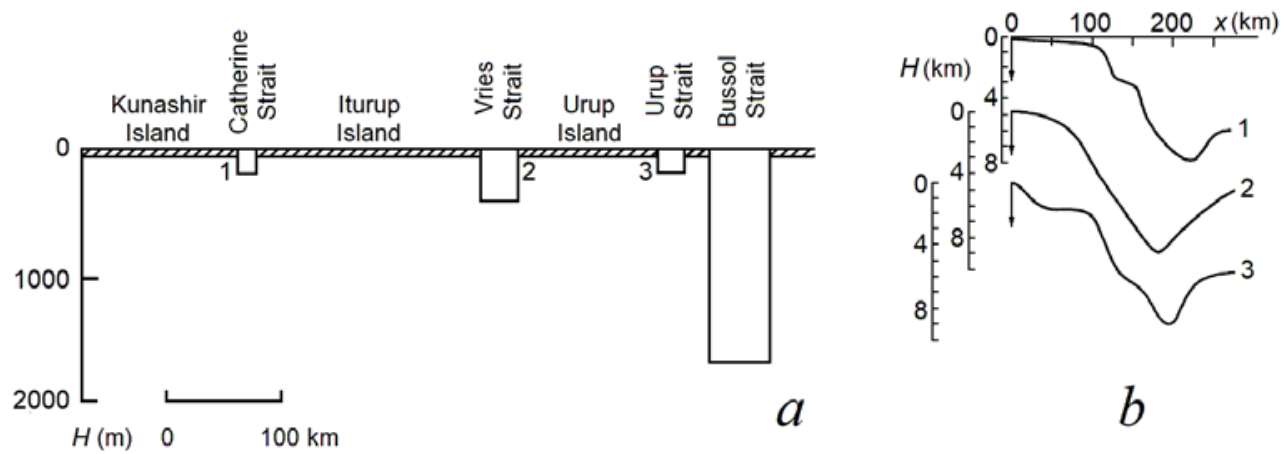

F i g. 4. A model of the ocean shelf with a straight shore line with straits [16, p. 229] (a) and transverse depth profiles for the shelf of the southern Kuril Islands [17, p. 87] (b)

Due to the obvious relationship between the generation time of wave packets and the beginning of the tide, the spectrum was calculated for sea level fluctuations with periods from 1 to 65 h, i.e., including the range of tidal fluctuations (Fig. 5). The tidal waves $K_{1}$ and $K_{2}$ are clearly seen there, but the appearance of wave packets in the area of Cape Castricum is associated only with the diurnal $K_{1}$ tide.

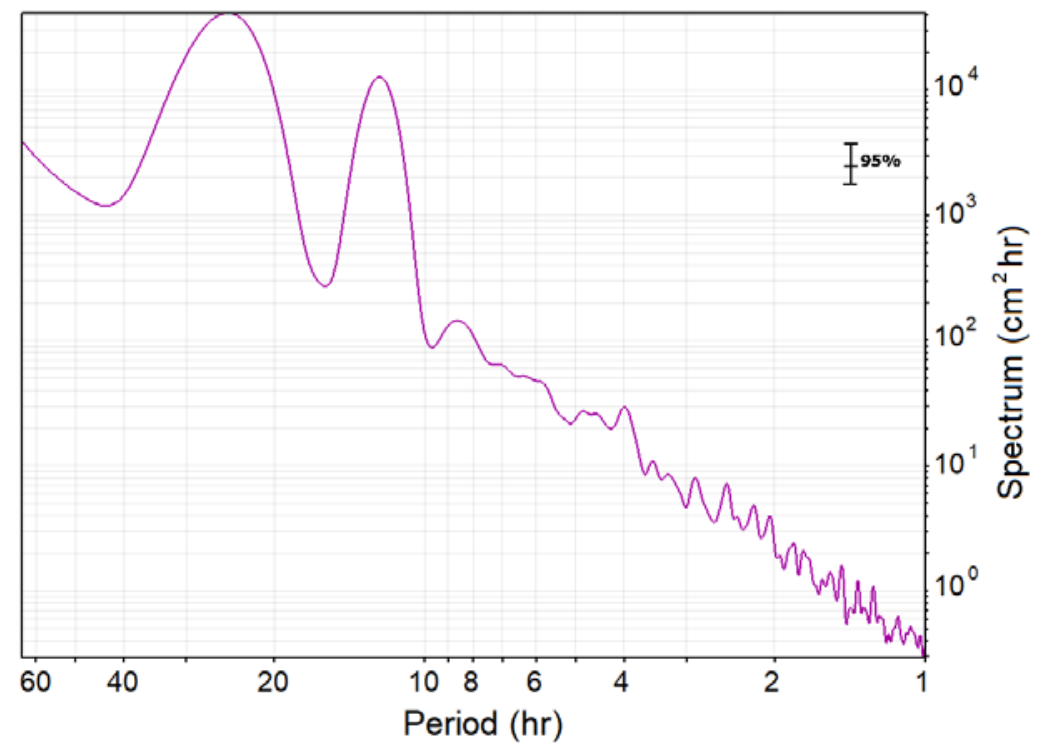

F i g. 5. Spectrum of tidal fluctuations of the level at the instrument's location near Cape Castricum calculated from the 3.7-month-long time series

It is known that when a flow goes around elongated cylindrical bodies (or other linearly elongated profiles), with a longitudinal axis perpendicular to the flow direction, it can detach from the body. (This is true for both laminar and turbulent flows [10].) As a result of flow separation, the vortices periodically separate from a point that is located at different distances from the beginning of the flow for laminar and turbulent flow: when the flow is laminar, it is located PHYSICAL OCEANOGRAPHY VOL. 28 ISS. 1 (2021) 
upstream. At Reynolds numbers between 100 and 200, the flow is unstable and vortices are separated in an alternating sequence [10]. Earlier studies of the nearbottom boundary layer at a depth of about $85 \mathrm{~m}$ in the South Kuril Islands show that the turbulent regime of tidal currents prevails, except at short periods of up to 30-50 min, when the current reverses [18].

The authors believe that for the situation identified, the tidal $K_{1}$ flow of the wave cyclically breaks away from the elevation in the Urup Strait located between the islands. This results in the formation of vortices, which cause the initiation of the wave packets observed. In this case, the periods of the detached vortices determine the periods of the waves in the enveloping soliton packet, i.e., the modulation.

The hypothesis above is confirmed by the arguments presented in [8] and by the results of studies carried out in [7]. Apel considers the generation of solitary wave packets by a tidal flow over shallow rock-bars or shores and remarks that the appearance of these packets is synchronized with the semidiurnal $K_{2}$ tide. Nakamura and co-authors also present the results of a study of the nonstationary wave generation by the diurnal $K_{1}$ tide in the Bussol Strait, adjacent to the Urup Strait.

However, the authors of [7] and [8] consider the generation of internal single waves. In the case being investigated here, the authors believe that the identified waves are barotropic and not internal, since the waves discussed herein are detected by seafloor-mounted pressure sensors and have substantially larger amplitudes than those expected for internal waves at the locations of the devices [19].

The generation of waves due to a flow around an obstacle is also well known in meteorology. In [20], it is argued that if the air is forced to cross a mountain range in statically stable conditions, then the air sections start to oscillate about their equilibrium positions. This leads to the wave system formation downwind of the mountain.

Structure of wave packets. The nature of the wave packets is considered in this section. Fig. 6 presents detailed unfiltered and filtered data fragments containing wave packets, to identify probable oceanographic mechanisms that could lead to oscillations with a period of $1.6 \mathrm{~h}$ that are located within each envelope of the wave packet. Wave periods of 1.6 hours can be associated with Poincaré or marginal waves, as well as with shelf seiches.

The calculation of the amplification factor of Poincaré waves is made using the equation taken from [16, p. 39], for a convex exponential profile with approximation $h(x)=-e^{0.63 x}$, where $x$ is the distance from the coast in kilometers. This shows that, for a given bottom topography, amplification of Poincaré waves with amplitudes exceeding the background level and with periods of about $40 \mathrm{~min}$ or less is possible. Since the waves recorded in the present study have a period of about 1.6 hours, this means that the recorded waves cannot be Poincaré waves. 

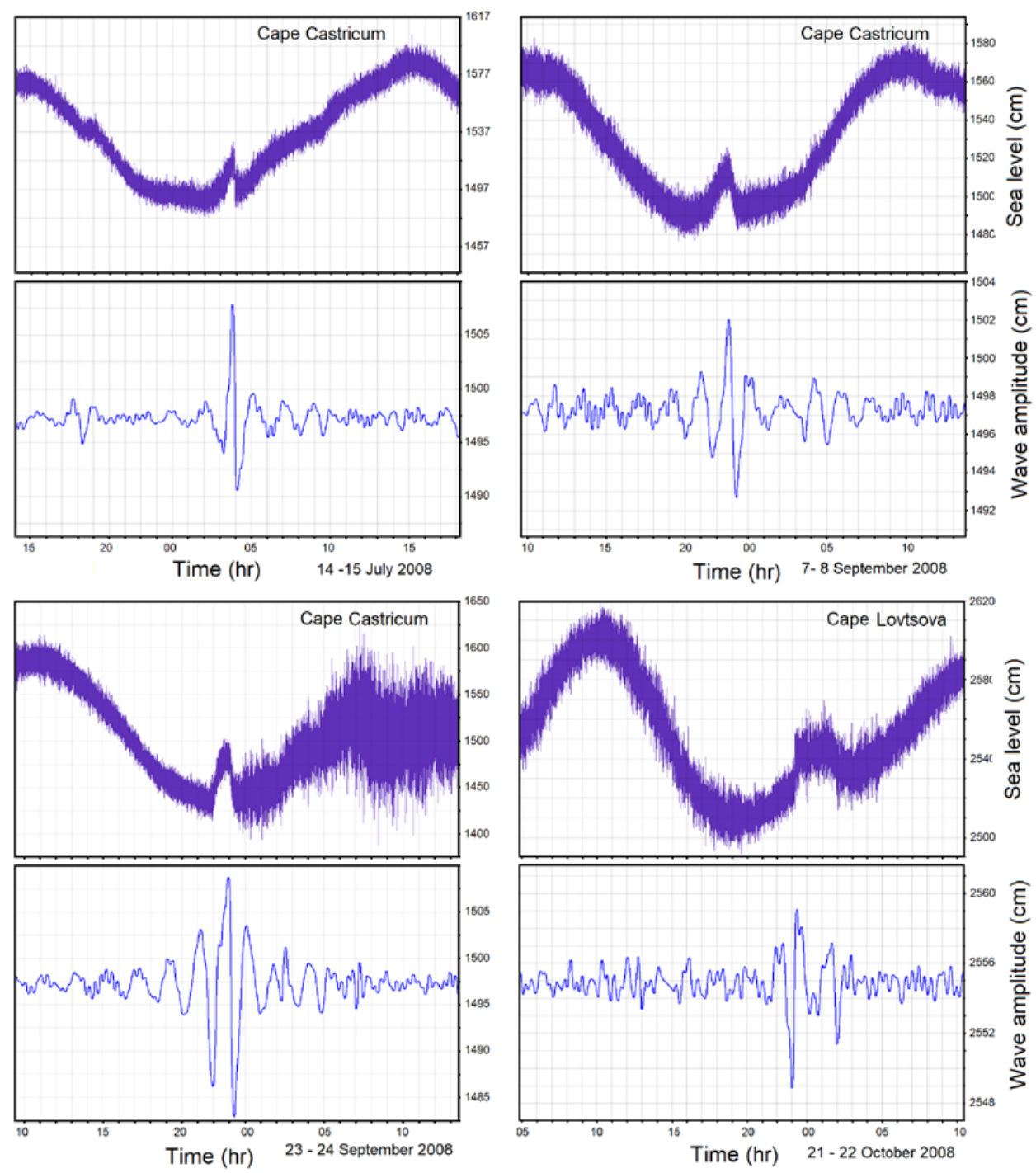

F i g. 6. Time series segments and the same segments after their processing by the filter with a passband from 20 minutes to 2.5 hours

To test the possibility that shelf seiches are being generated at a period of $1.6 \mathrm{~h}$, the theoretical periods of shelf seiches were calculated for the bottom slope in the area of Cape Castricum $(\alpha=0.021)$ and the values of the width of the first shelf $(\mathrm{L} \approx 95 \mathrm{~km})$, according to those given in [17, p. 102; 21] by the formula

$$
T_{n}=8 \sqrt{L} /(n \sqrt{g \alpha}),
$$

where $n=1,3,5, \ldots$ is the mode number. Calculations show that the first mode period of the shelf seiche is about $1.5 \mathrm{~h}$, i.e., only slightly less than the period of the detected fluctuations of $1.6 \mathrm{~h}$. 
At the same time, the results of the study [22] showed that the amplitude of such seiches decreases slowly and that, as a result, large amplitudes of sea seiches are recorded for a long time after excitation. This is inconsistent with the picture shown in Fig. 6. It can be seen that the attenuation of oscillations with a period of $1.6 \mathrm{~h}$ occurs quickly and their heights outline the envelope of a single wave. On the other hand, in [13] it is concluded that there is a relation between waves of the soliton type and the rise in the energy of oscillations in the sea, consisting of waves with the same period as those discussed here.

The possibility of generating edge waves with observable periods was also studied using the results of $[16,17]$. The actual profile corresponding to the data collection site near Cape Castricum is presented as profile 3 in Fig. 4, taken from [17, p. 87]. The dispersion diagram corresponding to this profile, also taken from [17, p. 91], shows the possibility of the existence of edge waves with a length of about $11.300 \mathrm{~m}$ with a period of $1.6 \mathrm{~h}$. The coincidence of the values of the measured wave periods and periods obtained from the theoretical model suggests that the waves detected at Cape Castricum are most likely to be edge modes.

Thus, in the Cape Castricum area, the resonance features of the water domain make possible the coexistence of two types of waves. And that, as a point of fact, waves with periods close to 1.6 hours can emerge inside single wave packets.

A simple statistical method developed for detecting the so-called anomalous waves [23] was also implemented using two-day filtered time series recorded at the Cape Castricum and Cape Lovtsova sites. General statistical characteristics were calculated using the following standard formulae

$$
\begin{gathered}
\bar{z}=\frac{1}{N} \sum_{i=1}^{N} z_{i}, \\
\sigma=\sqrt{\frac{1}{N} \sum_{i=1}^{N}\left(z_{i}-\bar{z}\right)^{2},} \\
S k=\frac{1}{N \sigma^{3}} \sum_{i=1}^{N}\left(z_{i}-\bar{z}\right)^{3}, \\
K u=\frac{1}{N \sigma^{4}} \sum_{i=1}^{N}\left(z_{i}-\bar{z}\right)^{4},
\end{gathered}
$$

where $N$ is the quantity of measurements in the interval (about 172800); $z_{i}$ is the sea level value, measured from the bottom according to the hydrostatic formula for pressure; $\bar{z}$ is the mean sea level value in a 2-day interval; $S k$ is the coefficient of skewness and $K u$ is the coefficient of kurtosis.

Data and results of calculations, including the date of the beginning of each 2day time series, are given in the table, which shows that the calculated statistical characteristics demonstrate significant variability over 115 days, whilst $\sigma$ is larger for segments of the time series containing wave packets. 
For background time series that do not contain wave packets, the average value of the rms deviation is $1.90 \mathrm{~cm}$, the coefficient of skewness is 0.01 and kurtosis is 3.36. These values differ little from Gaussian ( $S k=0$ and $K u=3$ ), so that the background disturbance in the range of periods from $20 \mathrm{~min}$ to $2.5 \mathrm{~h}$ in the absence of wave packets and between them is close to Gaussian.

For time series segments containing wave packets, the mean value of the standard deviation is $3.93 \mathrm{~cm}$, which is twice the background level value. In these cases, the kurtosis value is also significantly different from Gaussian, the average value of which is 12.53 . At the same time, a small negative value of the coefficient of skewness indicates the presence of left-sided asymmetry, and a larger value of the coefficient of kurtosis (in comparison with the Gaussian) indicates a greater probability of the appearance of abnormally large waves [23]. When wave packets appear, wave field stationarity is significantly violated.

We review which of the observed waves are above the background level and can hence be said to be anomalous. Since the background waves are Gaussian when wave packets are absent in the time series, as shown above, then a significant amplitude can be recognized by the expression $A_{s}=2 \sigma$, given in [23, p. 37]. A wave will be defined as abnormal if its amplitude $A_{a b}>2 A_{s}=4 \sigma$.

The table shows calculated $A_{a b}$ and $A_{s}$ results. It can be seen that a significant wave amplitude varies from case to case and its average value is $3.81 \mathrm{~cm}$. Waves with an amplitude of more than $7.62 \mathrm{~cm}$ will be considered abnormal. All the cases considered from the observed time series fragments shown in Fig. 6 after band pass filtering meet this criterion. For the fragment dated July 14-15, the maximum wave amplitude is about $17.5 \mathrm{~cm}$ and, for the fragment dated September 23-24, it is about $25 \mathrm{~cm}$; both values exceed the critical value of the anomaly criterion. In the other two cases, the amplitudes are 9 and $10 \mathrm{~cm}$, i.e., they are almost one and a half times higher than the background level in the considered range of periods and also correspond, albeit with a smaller margin, to the criterion of anomalous waves that is used in the present study.

Model and analysis. The notion of using the Korteweg - de Vries equation for the interpretation of the observed wave packets was considered. Wave fragments in normalized coordinates of time and amplitude were constructed for the four detected soliton-type waves. For the $\mathrm{KdV}$ equation, the following coordinates are used: $t \sqrt{A}$, where $t$ is time and $A$ is the wave amplitude; and $\eta / A$, where $\eta$ is the elevation of the surface. Such time series normalization permits profiles to be compared with the sech ${ }^{2}$ function since, without normalization, the sea surface elevation and the soliton duration depend on its amplitude.

The graph in Fig. 7 shows waveforms (time series fragments) of the KdV equation. There, all four curves in normalized coordinates are folded into one, affirming that the KdV equation is the preferred model for describing the detected waves. Consequently, the KdV equation applied to long unidirectional gravity waves in water was used to simulate surface waves on a fluid [24] and, thereby, to synthesize our results. 


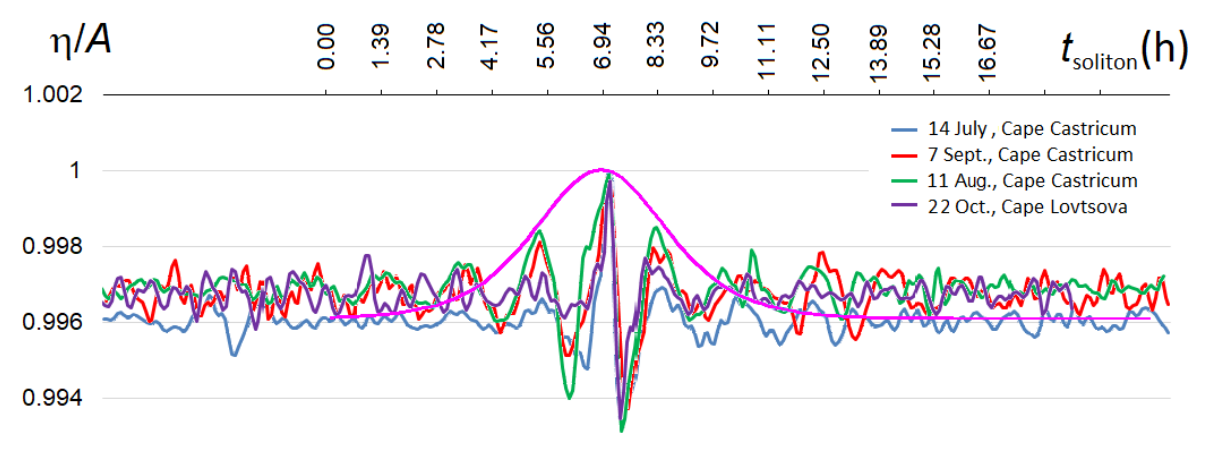

0.992

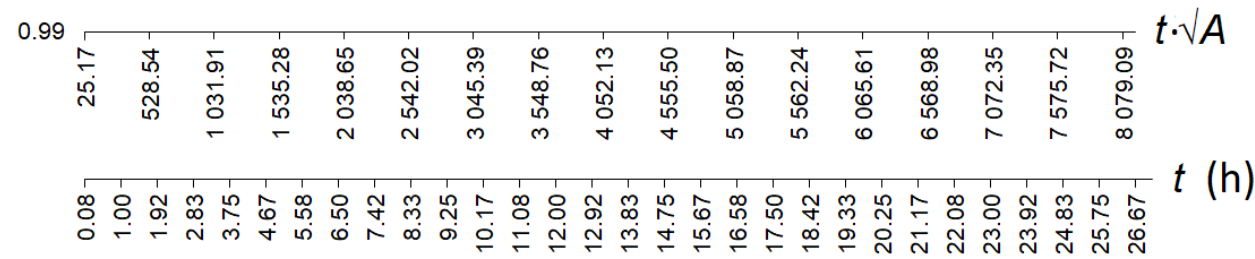

F i g. 7. Waveforms in the normalized time and amplitude coordinates for the model according to the KdV equation (pink line). The lower horizontal scale shows time in hours for the wave with the start of its record on June 14, 2008. The upper time scale refers to the soliton

The classical solution of the Korteweg - de Vries equation. The time form of the KdV equation [14] is more useful to describe the spatial evolution of the data measured at the point $x_{0}$, i.e., the boundary value problem. This equation is denoted as the TKdV equation and written

$$
\frac{\partial \eta}{\partial x}+\frac{1}{c_{0}} \frac{\partial \eta}{\partial t}-\alpha \eta \frac{\partial \eta}{\partial t}-\beta \frac{\partial^{3} \eta}{\partial t^{3}}=0
$$

where $c_{0}=\sqrt{g h ;} \alpha=3\left(2 c_{0} h\right) ; \beta=h^{2}\left(6 c_{0}^{3}\right) ; g$ is the acceleration due to gravity and $h$ is the undisturbed water depth.

The single soliton solution of the TKdV equation (1) has the following form [12, p. 3]

$$
\eta(x, t)=\operatorname{Asech}^{2}\left(\frac{t-x V}{T}\right)
$$

where velocity $V$ and duration $T$ depend on the amplitude $A$ :

$$
V=\frac{c_{0}}{1-c_{0} \alpha A / 3} \approx c_{0}\left(1-\frac{c_{0} \alpha A}{3}\right), T=\sqrt{\frac{12 \beta}{\alpha A}} .
$$


The approximate (binomial) expression for $V$ in equation (3) is valid for smallamplitude solitons as long as $c_{0} \alpha A / 3<<1$.

Using equations (2) and (3) for the values of the wave depth and amplitude obtained from field data, a graph of the sea surface elevation and the profile of the actually observed single wave were constructed (pink curve in Fig. 7). The following parameter values were used in the calculations: $A=0.125 \mathrm{~m}$; $h=15 \mathrm{~m} ; x=60 \times 10^{3} \mathrm{~m}$, and the duration of the wave packet $\mathrm{T}=8000$ s, i.e., $T \sqrt{A}=2828 \mathrm{~m}^{1 / 2} \mathrm{~s}$ during normalization. It is evident that the pink soliton curve in Fig. 7 encompasses the wave packet captured by the IMGiG FEB RAS.

Harmonic breathers. The presence of 1.6-hour oscillations within the envelope of single wave packets, i.e., modulation, suggests that the well-known classical solution of the KdV equation [12] is incomplete and that further research is needed. On the other hand, the $\mathrm{KdV}$ equation is non-linear and, although researchers have made significant progress in solving it, invoking the inverse scattering transform (IST) as an alternative to a linear Fourier transform remains challenging. The procedure for solving the TKdV equation using the Miura transformation [25] involves: a) solving the forward scattering problem at $x=0$ using the Schrödinger equation with the potential $\eta(x, t)$, that is, $-\partial^{2} \chi / \partial t^{2}+\eta \chi=$ $=\lambda^{2} \chi$, to find a finite number of discrete eigenvalues $\lambda_{n}$ and a continuous spectrum of eigenvalues $\lambda$; b) finding out how the scattering data evolve spatially, i.e., with a change in $x$; c) calculating $\eta(x, t)$ at position $x$ using scattering data in $x$, and then solving an integral equation with a kernel consisting of a superposition of discrete wave modes $\left(i \lambda_{n}\right)$ and a continuous spectrum of wave modes $(i \lambda)$, and differentiating the result with respect to $t$ [26]. Each step of the IST, i.e., "a to c", leads to a unique solution to the KdV equation that consists of two components: a part that is obtained from $N$ discrete eigenvalues that maps to $N$ solitons and a part corresponding to the continuous spectrum. For this study, which is primarily concerned with understanding oceanographic observational data, it would be very difficult to establish a convincingly strong connection between mathematical theory and observational data. Despite this, the modulation within each single wave packet cannot be ignored, since it is an important and very noticeable feature of the recorded time series in the presence of groups of single wave packets.

The author of $[3,4]$ briefly explains the IST application for solving the spatial $\mathrm{KdV}$ equation, but then considers the continuous spectral part (soliton-free) which is a superposition of linear harmonics. For two harmonics corresponding to the frequency $\pm \lambda$ (wave number for TKdV), he writes down an elemental solution for $\eta(x, t)$, which he calls a harmonic breather [3, p. 5118]. Then, by analogy with solitons, Kovalev defines a nonlinear superposition of $N$ harmonic breathers with $N$ different frequencies (wave numbers) $\Lambda=\left\{\lambda_{1}, \lambda_{2}, \ldots, \lambda_{N}\right\}$ and, potentially, $N$ different displacements and phases. He argues that the finite sum of nonlinear harmonic breathers is similar to the sum of the harmonics of the Fourier transform, interpreting various functions as linear superpositions of trigonometric functions. 


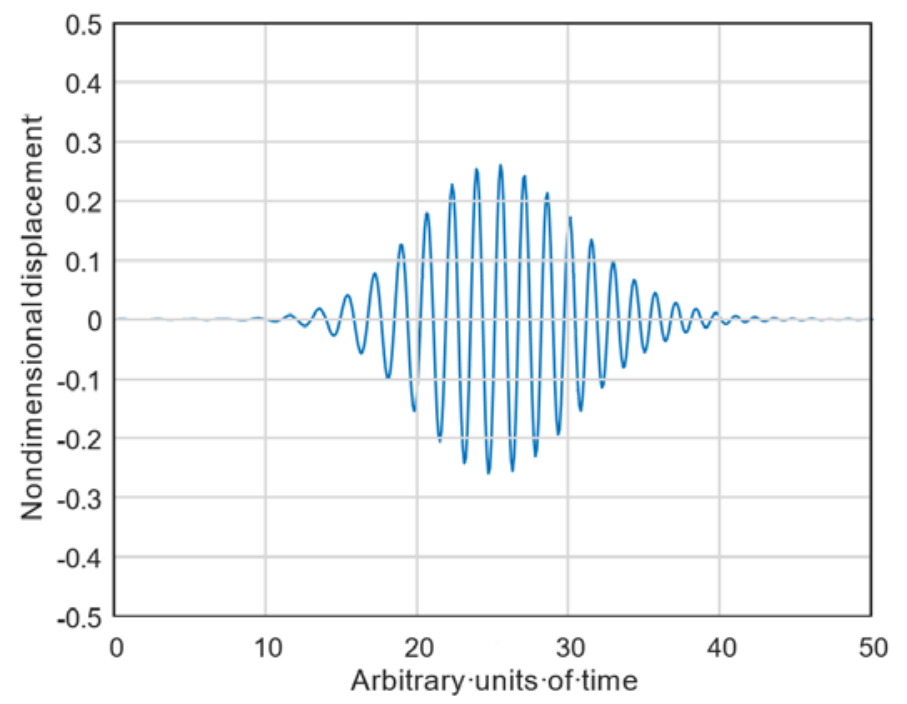

F i g. 8. Wave packet generated by 21 harmonic breathers at nondimensional position $x=-0.525$ (the figure replicates the bottom right subplot of Fig. 3 from [3, p. 5121], the first figure was obtained using the TKdV equation and the second one - the KdV equation)

A sample of a wave packet shown in Fig. 8 was calculated using the same parameters as in Fig. 3 from [3], but with interchangeable $x$ and $t$ so that we can effectively work with a sort of physically artificial version of the TKdV equation, i.e., the wave packet shown is the observational data that could be obtained on a device located at a distance of 0.525 dimensionless units from $x=0$, measured in the negative direction.

Fig. 8 is shown not to simulate one of the wave packets from the dataset, but as a qualitative demonstration of the possibility of creating modulation within the external single envelope wave using a superposition of harmonic breathers. The inclusion of additional harmonic breathers outside of $N=21$ has little effect on the wave packet and only adds confidence that the summation emulates a continuous spectral integral.

Conclusions. To determine wave processes in the coastal zone of the islands, an analysis of wave data recorded in 2008-2009 was carried out near Cape Lovtsova (Kunashir Island), and Capes Van der Lind and Castricum (Urup Island). The observations were carried out using autonomous bottom-mounted ARW pressure sensors, which record over a wide range of wave periods stretching from $2 \mathrm{~s}$ to tidal periods. The duration of the recorded time series varied from 4 to 6 months.

Preprocessing of the recorded time series using a $20 \mathrm{~min}$ to $2.5 \mathrm{~h}$ bandwidth filter showed several distinct wave packet solitons in the vicinity of Cape Castricum and Cape Lovtsova, which enclosed oscillations at a period of about $1.6 \mathrm{~h}$. These wave packets appear in groups of approximately six waves, synchronous with the start of the $K_{1}$ tide for Cape Castricum, and it is observed that the $K_{1}$ wave dominates in the calculated spectrum of tidal oscillations. Spectral analysis carried out for 4.5-day-long time series in the presence of a group of 
solitons or their absence showed that a wave energy increase is observed in the interval of periods from 0.5 to $5.5 \mathrm{~h}$ when solitons appear.

It was found that the sea state does not affect the generation of wave packets, but the water depth in the strait is of great importance: wave packets appear near the capes in the rather shallow straits of Catherine and Urup but are not observed in deeper straits. It is assumed that these processes may also be affected by the coastal shelf projection, which is clearly visible on the depth profiles (Fig. 4, b).

Based on the results obtained and the study of literary sources, it can be assumed that wave packets are generated when a tidal current flows around an elevation in the Urup Strait between the islands. In this case, a cyclical separation of the tidal flow of the $K_{1}$ wave occurs with the formation of vortices, which, in turn, leads to the generation of the observed wave packets.

To determine abnormal waves in the time series, conventional statistical methods were used using 2-day fragments. It was found that background oscillations in the period range from $20 \mathrm{~min}$ to $2.5 \mathrm{~h}$ are close to Gaussian in the absence of wave packets, but the stationarity is violated when they are present. Using a simple amplitude criterion, the waves detected in the wave packets were deemed anomalous.

By transforming the time series into normalized time and amplitude coordinates, it was found that all examples of anomalous wave packets can be simulated using the $\mathrm{KdV}$ time equation. The classical solution $\eta=A \operatorname{sech}^{2}[(t-x / V) / T]$ replicated the wave packet envelope when the measured values of the water depth and amplitude are introduced, providing confidence that the observed wave packets are solitons. By superposing a set of harmonic breathers [3], the authors were able to illustrate qualitatively the presence of internal modulation within the observed solitons.

\section{REFERENCES}

1. Peregrine, D.H., 1983. Water Waves, Nonlinear Schrödinger Equations and Their Solutions. The ANZIAM Journal, 25(1), pp. 16-43. https://doi.org/10.1017/S0334270000003891

2. Jaworski, M., 1984. Breather-Like Solution of the Korteweg-de Vries Equation. Physics Letters A, 104(5), pp. 245-247. https://doi.org/10.1016/0375-9601(84)90060-4

3. Kovalyov, M., 1998. Modulating Properties of Harmonic Breather Solutions of KdV. Journal of Physics A: Mathematical and General, 31(22), pp. 5117-5128. https://doi.org/10.1088/0305-4470/31/22/012

4. Kovalyov, M., 2005. On a Class of Solutions of KdV. Journal of Differential Equations, 213(1), pp. 1-80. https://doi.org/10.1016/j.jde.2003.07.016

5. Akhmediev, N., Ankiewicz, A. and Taki M., 2009. Waves That Appear from Nowhere and Disappear without a Trace. Physics Letters A, 373(6), pp. 675-678. https://doi.org/10.1016/j.physleta.2008.12.036

6. Chabchoub, A., Mozumi, K., Hoffmann, N., Babanin, A.V., Toffoli, A., Steer, J.N., van den Bremer, T.S., Akhmediev, N., Onorato, M. and Waseda, T., 2019. Directional Soliton and Breather Beams. Proceedings of the National Academy of Sciences, 116(20), pp. 9759-9763. https://doi.org/10.1073/pnas.1821970116

7. Nakamura, T., Awaji, T., Hatayama, T., Akitomo, K., Takizawa, T., Kono, T., Kawasaki, Y. and Fukasawa, M., 2000. The Generation of Large-Amplitude Unsteady Lee Waves by Subinertial $K_{1}$ Tidal Flow: A Possible Vertical Mixing Mechanism in the Kuril Straits. Journal of Physical Oceanography, 30(7), pp. 1601-1621. https://doi.org/10.1175/15200485(2000)030\%3C1601:TGOLAU\%3E2.0.CO;2 
8. Apel, J.R., 2004. Oceanic Internal Waves and Solitons. In: C. R. Jackson and J. R. Apel, Eds., 2004. Synthetic Aperture Radar Marine User's Manual. Washington, DC.: U.S. Department of Commerce, Chapter 7, pp. 189-206. Available at: https://www.sarusersmanual.com/ManualPDF/NOAASARManual_CH07_pg189-206.pdf [Accessed: 10 November 2020].

9. Apel, J.R., Ostrovsky, L.A., Stepanyants, Y.A. and Lynch, J.F., 2006. Internal Solitons in the Ocean. Woods Hole, MA: Woods Hole Oceanographic Institution, 108 p. doi:10.1575/1912/1070

10. Chang, P.K., 1970. Separation of Flow. Oxford, UK: Pergamon, 796 p. https://doi.org/10.1016/C2013-0-05541-7

11. Korteweg, D.J. and de Vries, G., 1895. XLI. On the Change of Form of Long Waves Advancing in a Rectangular Canal, and on a New Type of Long Stationary Waves. The London, Edinburgh, and Dublin Philosophical Magazine and Journal of Science, 39(240), pp. 422-443. https://doi.org/10.1080/14786449508620739

12. Giovanangeli, J.-P., Kharif, C. and Stepanyants, Y.A., 2018. Soliton Spectra of Random Water Waves in Shallow Basins. Mathematical Modelling of Natural Phenomena, 13(4), 40. https://doi.org/10.1051/mmnp/2018018

13. De Jong, M.P.C., 2004. Origin and Prediction of Seiches in Rotterdam Harbour Basins: doctoral thesis. Delft, The Netherlands: Delft University of Technology, 119 p. Available at: http://resolver.tudelft.nl/uuid:d7ce7779-bf81-47b7-bc14-e01ce5e6856b [Accessed: 10 November 2020].

14. Osborne, A.R., Segre, E., Boffetta, G. and Cavaleri, L., 1991. Soliton Basis States in ShallowWater Ocean Surface Waves. Physical Reviews Letters, 67(5), pp. 592-595. https://doi.org/10.1103/PhysRevLett.67.592

15. Costa, A., Osborne, A.R., Resio, D.T., Alessio, S., Chrivì, E., Saggese, E., Bellomo, K. and Long, C.E., 2014. Soliton Turbulence in Shallow Water Ocean Surface Waves. Physical Reviews Letters, 113(10), 108501. https://doi.org/10.1103/PhysRevLett.113.108501

16. Efimov, V.V., Ed., 1985. Waves in the Border Regions of the Ocean. Leningrad: Gidrometeoizdat, 280 p. (in Russian).

17. Rabinovich, A.B., 1993. Long Gravitational Waves in the Ocean: Capture, Resonance, and Radiation. Saint Petersburg: Gidrometeoizdat, 325 p. (in Russian).

18. Kovalev, P.D. and Rabinovich, A.B., 1980. Measurements of Bottom Tidal Currents in the Vicinity of the Kuril-Kamchatka Arc. Oceanology, 20(3), pp. 451-458.

19. Thomas, J.A., Lerczak, J.A. and Moum, J.N., 2016. Horizontal Variability of High-Frequency Nonlinear Internal Waves in Massachusetts Bay Detected by an Array of Seafloor Pressure Sensors. Journal of Geophysical Research: Oceans, 121(8), pp. 5587-5607. https://doi.org/10.1002/2016JC011866

20. Zdunkowski, W. and Bott, A., 2003. Dynamics of the Atmosphere: A Course in Theoretical Meteorology. Cambridge, UK: Cambridge University Press, 719 p. https://doi.org/10.1017/CBO9780511805462

21. Rabinovich, A.B., 2009. Seiches and harbor oscillations. In: Young C. Kim, Ed., 2009. Handbook of Coastal and Ocean Engineering. Singapore: World Scientific, pp. 193-236. https://doi.org/10.1142/9789812819307_0009

22. Kovalev, P.D. and Kovalev, D.P., 2013. Seiche Vibrations Features in Bays and Inlets of Far East: Primorye, Sakhalin, Southern Kuril Islands. Tambov University Reports. Series: Natural and Technical Sciences, 18(4), pp. 1377-1382. Available at: http://journals.tsutmb.ru/go/18100198/2013/4/1377-1382/ [Accessed: 10 November 2020] (in Russian).

23. Zaitsev, A., Malashenko, A., Kostenko, I., Pelinovsky, E. and Kuznetsov, K., 2012. Freak Waves Registration in the Aniva Bay, Okhotsk Sea. Transactions of Nizhni Novgorod State Technical University n. a. R.Y. Alexeev, (1), pp. 33-41. Available at: https://www.nntu.ru/frontend/web/ngtu/files/nauka/izdaniya/trudy/2012/01/2012-01.pdf [Accessed: 10 November 2020] (in Russian). 
24. Cazaubiel, A., Michel, G., Lepot, S., Semin, B., Aumaître, S., Berhanu, M., Bonnefoy, F. and Falcon, E., 2018. Coexistence of Solitons and Extreme Events in Deep Water Surface Waves. Physical Reviews Fluids, 3(11), 114802. doi:10.1103/PhysRevFluids.3.114802

25. Miura, R.M., 1968. Korteweg-de Vries Equation and Generalizations. I. A Remarkable Explicit Nonlinear Transformation. Journal of Mathematical Physics, 9(8), pp. 1202-1204. https://doi.org/10.1063/1.1664700

26. Ablowitz, M.J., 1978. Lectures on the Inverse Scattering Transform. Studies in Applied Mathematics, 58(1), pp. 17-94. https://doi.org/10.1002/sapm197858117

About the authors:

Vernon A. Squire, Professor Emeritus, Department of Mathematics \& Statistics, University of Otago (Dunedin 9054, New Zealand), ORCID ID: 0000-0002-5570-3446, SCOPUS Author ID: 7004257488, vernon.squire@otago.ac.nz

Peter D. Kovalev, Leading Research Associate, Institute of Marine Geology and Geophysics, Far Eastern Branch of Russian Academy of Sciences (1b Nauki Str., Yuzhno-Sahalinsk, 693022, Russian Federation), Dr. Sci. (Engineer.), ORCID ID: 0000-0002-7509-4107, SCOPUS Author ID:16429135400, ResearcherID: V-8662-2018, p.kovalev@imgg.ru

Dmitry P. Kovalev, Leading Research Associate, Institute of Marine Geology and Geophysics,

Far Eastern Branch of Russian Academy of Sciences (1b Nauki Str., Yuzhno-Sahalinsk, 693022, Russian Federation), Dr. Sci. (Phys.-Math), ORCID ID: 0000-0002-5184-2350, SCOPUS AuthorID: 26032627700, ResearcherID: A-9300-2016, d.kovalev@imgg.ru

Contribution of co-authors:

Vernon A. Squire - methodology, conceptualization, investigation, software, formal analysis, writing - original and final drafts, writing - review \& editing

Peter D. Kovalev and Dmitry P. Kovalev - conceptualization, translation activities, methodology, data curation, formal analysis, funding acquisition, software, investigation, methodology

All the authors have read and approved the final manuscript.

The authors declare that they have no conflict of interest. 